\title{
DETERMINATION OF THE FREQUENCY DISPERSION REGION OF THE TRANSPORT COEFFICIENTS OF CLASSICAL LIQUIDS DEPENDING ON THE CHARACTER OF ATTENUATION OF RELAXING FLOWS
}

\section{S. ODINAEV}

PACS 66.20.Cy

(C) 2011
M. Osimi Tajik Technical University

(10, Academicians Rajabovs Str., Dushanbe 734042, Tajikistan; e-mail: odsb@tarena-tj. org)

\begin{abstract}
We consider the frequency dispersion region of the dynamic shear viscosity coefficient $\eta_{s}(\omega)$ of simple liquids obtained by the method of kinetic equations, where the equilibrium structure of a liquid is restored according to the diffusion law or exponentially. At a certain choice of the intermolecular interaction potential $\Phi(|\mathbf{r}|)$ and the equilibrium radial distribution function $g_{0}(|\mathbf{r}|)$, the coefficient $\eta_{s}(\omega)$ for liquid argon was numerically calculated as a function of the density $\rho$, temperature $T$, and frequency $\omega$. The obtained theoretical values of the shear viscosity $\eta_{s}(\omega)$ are in a satisfactory quantitative agreement with experimental data. It is shown that the frequency dispersion region of $\eta_{s}(\omega)$ obtained on the basis of the diffusive mechanism, i.e. structural relaxation, is large $\left(\sim 10^{5} \mathrm{~Hz}\right)$. In the case of the exponential attenuation of the viscous stress tensor, this region is narrow $\left(\sim 10^{2} \mathrm{~Hz}\right)$, which agrees both with acoustic measurements and the results of a phenomenological theory.
\end{abstract}

\section{Statement of the Problem}

Investigations of the dynamic transport coefficients and the corresponding dynamic moduli of elasticity in a wide frequency range provide the important information about the kinetics of irreversible processes in liquids, and their molecular structure, their relation with intermolecular phenomena, as well as the mechanism of restoration of their equilibrium structure.

The viscous flow of a liquid is accompanied by the mass, momentum, and energy transfer from its one region to another one, which results in transport phenomena characterized by the diffusion, viscosity, and heat conductivity coefficients. The rates of transport processes in liquids are high; in the states close to the equilibrium one, they are proportional to the mass, momentum, and temperature gradients. It is worth noting that, at high deformation rates in liquids [1], they acquire elastic properties along with the viscous flow. Moreover, each kind of transport is related to definite elastic properties. In this case, the viscoelastic properties of liquids can be studied using the Kelvin or Maxwell mechanical model, whereas the rheology equation will be the Kelvin (Maxwell) or Voigt equation, whose resulting stress consists of the elastic component proportional to the deformation of the liquid and the viscous component proportional to the deformation rate [2]. In this case, the acoustic equations include a complex effective modulus of elasticity (for example, formula (6.30) in [2]), where the real part represents the dynamic modulus of elasticity and the imaginary one is the dynamic viscosity, i.e. they are frequency dependent functions.

According to [2, 3], the shear and bulk viscosities can have one or several characteristic relaxation times. Consequently, there can exist one or more Maxwell mechanisms of viscoelasticity (for example, formulas (6.30), (6.42) in [2] and (5.17) in [3]). Based on these formulas and Figs. 29 and 33, the cited works adduce the frequency dependences of the dynamic modulus of elasticity $E(\omega)$ and the dynamic viscosity $\eta(\omega)$ in the case of one and two relaxation processes with differing relaxation times $\left(\tau_{2}=(1 / 50) \tau_{1}\right)$. The frequency dependences $E(\omega)$ and $\eta(\omega)$ are depicted on the logarithmic scale and occupy an interval covering more than three decades. On the natural scale, their low-frequency part would be absolutely indistinguishable. If the relaxation times are close, the curves can appear monotonous, and their form would not allow one to judge if there actually exists one or two relaxation times (for example, Fig. 34 in [2] at $\left.\tau_{2}=(1 / 3) \tau_{1}\right)$, i.e. there is no point of inflection. According to $[2,3]$, with increasing width of the frequency dispersion region and in the absence of sufficiently pronounced steps on the curve, no conclusions can be made about individual relaxation mechanisms. The only justified method of interpreting such curves is, to the authors' mind, to introduce a continuous spectrum of relaxation times characteristic of the process of structural relaxation in liquids. In this case, the sums in the complex modulus of elasticity are replaced by integrals 
(formula (6.43) in [2] and formulas (5.47)-(5.54) in [3]). However, the densities of the relaxation time spectrum in the integrands of the functions $H(\tau), k\left(\tau / \tau^{\prime}\right)$, and $g\left(\tau / \tau^{\prime}\right)$ remain unknown. According to [3], the choice of these distribution functions is, to some degree, arbitrary, as an equally good agreement with experiment often can be achieved by using several functions. It is worth noting that all the above-analyzed analytic expressions for the modulus of elasticity $E(\omega)$, the viscosity coefficient $\eta(\omega)$, the velocity $C(\omega)$, and the coefficient of absorption $\alpha(\omega)$ of sound waves in liquids were obtained in $[2,3]$ in the framework of the phenomenological theory.

The dynamic transport coefficients and the dynamic moduli of elasticity of liquids can be investigated using both the phenomenological and molecular-kinetic theories. The phenomenological theory describes observed phenomena based on the relations between macroscopically measured quantities and uses methods of the thermodynamics of irreversible processes. However, it does not allow one to study the detailed mechanism of transport processes. The molecular theory provides a description of the mechanism of transport phenomena and makes an attempt to derive the laws of these processes based on the properties of liquids, the variation of their structure, and the nature of internal relaxation processes in them. The molecular-kinetic theory enables one to obtain the equations of generalized hydrodynamics that have the same form as the macroscopic hydrodynamic equations derived with the use of the phenomenological theory with the difference that the transport coefficients are functions of the space and time scales of hydrodynamic quantities [4]. Their Fourier transformation provides frequency-dependent analytical expressions for the transport coefficients and the moduli of elasticity, i.e. the susceptibility coefficients of liquids. Consequently, the molecular theory of transport phenomena in liquids supplements the results of the phenomenological theory providing the understanding of the mechanisms and the character of these processes.

Experimental studies of the structural relaxation in liquids [2] showed that the frequency dispersion region of the viscosity and absorption coefficients covers two to five decades that cannot be determined with the help of the phenomenological theory. Due to this fact, we try to use the single microscopic theory for studying the frequency dispersion region of the dynamic viscosity coefficients of simple liquids with the contributions of the translational and structural relaxations depending on the attenuation character of the momentum flux in the momentum and configuration spaces.
It is worth noting that the static and dynamic transport coefficients in liquids most suitable for experimental research are the coefficients of shear viscosity, heat and electrical conductivities, as well as the acoustic parameters such as the velocity and the absorption of sound waves in a wide range of thermodynamic state parameters and frequencies. In order to determine the frequency dispersion region, we concentrate on considering the dynamic coefficient of shear viscosity $\eta_{s}(\omega)$ of simple liquids, where the equilibrium structure of the liquid is recovered according to the diffusion law or exponentially. Choosing $\Phi(|\mathbf{r}|)$ and $g(|\mathbf{r}|)$, we numerically calculate $\eta_{s}(\omega)$ for simple liquids, by basing on the obtained analytical expressions. In the case of a satisfactory quantitative agreement of the numerical results obtained for $\eta_{s}(\omega)$ with the existing literature experimental data, we apply this model subsequently to the theoretical study of the viscoelastic and acoustic properties of classical liquids, i.e. the bulk viscosity $\eta_{V}(\omega)$, moduli of shear $\mu(\omega)$ and bulk $K(\omega)$ elasticity, velocity $C(\omega)$, and absorption coefficient $\alpha(\omega)$.

\section{Initial Equations and Determination of Dynamic Shear Viscosity}

To obtain an analytical expression for $\eta_{s}(\omega)$, we will use the microscopic definition of the stress tensor $\sigma^{\alpha \beta}\left(\mathbf{q}_{1}, t\right)$ of simple liquids [5]:

$\sigma^{\alpha \beta}\left(\mathbf{q}_{1}, t\right)=K^{\alpha \beta}\left(\mathbf{q}_{1}, t\right)+$

$+\frac{\sigma^{3}}{2} \int \frac{\partial \Phi_{12}(|\mathbf{r}|)}{\partial r} \frac{r^{\alpha} r^{\beta}}{r} n_{2}\left(\mathbf{q}_{1}, \mathbf{r}, t\right) d \mathbf{r}$

where $K^{\alpha \beta}\left(\mathbf{q}_{1}, t\right)=n k T \delta^{\alpha \beta}+k^{\alpha \beta}\left(\mathbf{q}_{1}, t\right)$ is the kinetic part of the momentum flux tensor representing the momentum moment of the one-particle distribution function $f_{1}\left(\mathbf{x}_{1}, t\right)$, whereas $n k T$ and $k^{\alpha \beta}\left(\mathbf{q}_{1}, t\right)$ are the kinetic parts of the nonequilibrium pressure and the viscous stress tensor, respectively. In addition, $\mathbf{r}=$ $\left(q_{2}-q_{1}\right) / \sigma$ is the reduced intermolecular distance, $\sigma$ is the molecular diameter, $n\left(\mathbf{q}_{1}, t\right)$ and $T\left(\mathbf{q}_{1}, t\right)$ are the local density and the temperature, respectively, $\Phi_{12}(|\mathbf{r}|)$ is the intermolecular interaction potential, $n_{2}\left(\mathbf{q}_{1}, \mathbf{r}, t\right)$ is the nonequilibrium binary density of particles in the configuration space that represents the momentum moment of the two-particle distribution function $f_{2}\left(\mathbf{x}_{1}, \mathbf{x}_{2}, t\right)$, $\mathbf{x}=\{\mathbf{q}, \mathbf{p}\}$, while $\mathbf{q}$ and $\mathbf{p}$ are the coordinates and momenta of particles, respectively. The kinetic equations for the one- and two-particle distribution functions with regard for the contributions of large-scale fluctuations 
were obtained in [5]. The right-hand sides of the equation for $f_{1}\left(\mathbf{x}_{1}, t\right)$ and $f_{2}\left(\mathbf{x}_{1}, \mathbf{x}_{2}, t\right)$ are the Fokker-Planck collision operators that provide the time irreversibility of these equations and allow one to describe dissipative processes in liquids. The left-hand sides of these equations include integral terms (Bogolyubov dynamic terms) that yield the contributions of large-scale fluctuations to the relaxation parameters and are caused by collective interactions in liquids.

Expression (1) implies that the relaxation $\sigma^{\alpha \beta}\left(\mathbf{q}_{1}, t\right)$ consists of the translational and structural parts. The translational component is described by the equation for the kinetic part of the viscous stress tensor $k^{\alpha \beta}\left(\mathbf{q}_{1}, t\right)$ and is characterized by one relaxation time, whereas the structural component is described by the equation for the binary density of particles in the configuration space $n_{2}\left(\mathbf{q}_{1}, \mathbf{r}, t\right)$ and is characterized by a continuous spectrum of relaxation times.

The equations for the kinetic part of the viscous stress tensor $k^{\alpha \beta}\left(\mathbf{q}_{1}, t\right)$ and the binary density $n_{2}\left(\mathbf{q}_{1}, \mathbf{r}, t\right)$ were derived in [5] with the use of the kinetic equations for $f_{1}\left(\mathbf{x}_{1}, t\right)$ and $f_{2}\left(\mathbf{x}_{1}, \mathbf{x}_{2}, t\right)$ having the following form in the case of the linear approximation and independent flows:

$$
\frac{\partial k^{\alpha \beta}\left(\mathbf{q}_{1}, t\right)}{\partial t}+2 n_{0} k T_{0}\left\{\frac{\partial \vartheta^{\alpha}\left(q_{1}, t\right)}{\partial q_{1}^{\beta}}\right\}=-\frac{1}{\tau} k^{\alpha \beta}\left(\mathbf{q}_{1}, t\right),
$$

$$
\frac{\partial n_{2}\left(\mathbf{q}_{1}, \mathbf{r}, t\right)}{\partial t}+\omega_{0} \hat{L} n_{2}\left(\mathbf{q}_{1}, \mathbf{r}, t\right)=F\left(\mathbf{q}_{1}, \mathbf{r}, t\right)
$$

where $n_{0}$ and $T_{0}$ are the equilibrium particle density and the temperature, respectively, $\vartheta^{\alpha}\left(\mathbf{q}_{1}, t\right)$ is the mean velocity of particles, $\tau=m /(2 \beta)$ is the characteristic time of translational relaxation (i.e, the time of the dispersal of $k^{\alpha \beta}\left(\mathbf{q}_{1}, t\right)$ in the momentum space $), \tau_{0}=\omega_{0}^{-1}=$ $\beta \sigma^{2} / 2 k T$ is the phenomenological parameter being an analog of the relaxation time of a diffusing molecule, $k$ is the Boltzmann constant, $\beta$ is the friction coefficient, and $\hat{L}=-\frac{\partial}{\partial r^{\alpha}}\left[\frac{\partial}{\partial r^{\alpha}}-\frac{\partial}{\partial r^{\alpha}} \ln g_{0}(|\mathbf{r}|)\right]$ is the Smoluchowski operator in the configuration space,

$$
\begin{aligned}
& F\left(\mathbf{q}_{1}, \mathbf{r}, t\right)=-2 n_{2}^{0}(|\mathbf{r}|)\left\{\left[1+\frac{1}{6} \frac{\partial \ln g_{0}(|\mathbf{r}|)}{\partial \ln r}-\right.\right. \\
& \left.-\frac{1}{2}\left[n\left(\frac{\partial \ln g_{0}(|\mathbf{r}|)}{\partial_{n}}\right)_{T}+\gamma T\left(\frac{\partial \ln g_{0}(|\mathbf{r}|)}{\partial_{T}}\right)_{n}\right]\right] \operatorname{div} \boldsymbol{\vartheta}+
\end{aligned}
$$

$\left.+\left(\frac{r^{\alpha} r^{\beta}-(1 / 3) r^{2} \delta^{\alpha \beta}}{r^{2}}\right) \frac{\partial \ln g_{0}(|\mathbf{r}|)}{\partial \ln r}\left\{\frac{\partial \vartheta^{\alpha}}{\partial q_{l}^{\beta}}\right\}\right\}$

$\left.\gamma=\left(n c_{V}\right)^{-1}(\partial P / \partial T)_{n} ; \quad n_{2}^{0}(|\mathbf{r}|)=n_{2}^{0} g_{0}^{(}|\mathbf{r}|\right)$ stands for the equilibrium binary density, $c_{V}$ is the isochoric heat capacity of a liquid, and $\left.g_{0}^{(}|\mathbf{r}|\right)$ is the equilibrium radial distribution function.

Equation (2) for $k^{\alpha \beta}\left(\mathbf{q}_{1}, t\right)$ represents an inhomogeneous linear differential equation with separating variables, whose solution can be easily presented in the exponential form with characteristic time $\tau$. Equation (3) represents an inhomogeneous parabolic equation, i.e. the Smoluchowski equation for the binary density $n_{2}\left(\mathbf{q}_{1}, \mathbf{r}, t\right)$ in the configuration space. The solution of Eq. (3) is a Cauchy problem $(t>0,-\infty \leq r \leq \infty)$ with the zero initial condition having the form [5]:

$n_{2}\left(\mathbf{q}_{1}, \mathbf{r}, t\right)=\int_{0}^{t} d t \int_{-\infty}^{-\infty} G\left(r, r_{1}, t-t_{1}\right) F\left(\mathbf{q}_{1}, \mathbf{r}_{1}, t_{1}\right) d \mathbf{r}_{1}$

where

$G\left(r, r_{1}, t-t_{1}\right)=\frac{2\left(r r_{1}\right)^{-1}}{(2 \pi)^{3}}\left[\frac{\pi}{\omega_{0}\left(t-t_{1}\right)}\right]^{1 / 2} \times$

$\times\left\{\exp \left[-\frac{\left(r-r_{1}\right)^{2}}{4 \omega_{0}\left(t-t_{1}\right)}\right]-\exp \left[-\frac{\left(r+r_{1}\right)^{2}}{4 \omega_{0}\left(t-t_{1}\right)}\right]\right\}$.

Expression (6) represents a fundamental solution (Green function) of the Smoluchowski equation (3) for $n_{2}\left(\mathbf{q}_{1}, \mathbf{r}, t\right)$ and describes the space-time behavior of the binary density and, consequently, the structural relaxation. As is seen from Eqs. (3)-(6), the process of reconstruction in classical liquids is of the diffusive character and can be described by a continuous spectrum of relaxation times. In this case, the dispersal of irreversible flows is diffusive and obeys the power law $t^{-d / 2}$ coinciding with the large-time behavior of autocorrelation functions [6-8].

Performing the time Fourier transformation in (1) and in the subsequent equations and using Eqs.(1), (2), (5), and (6), one obtains the dynamic coefficient of shear viscosity $\eta_{s}(\omega)[5,9]$ :

$\eta_{s}(\omega)=\frac{n k T \tau}{1+(\omega \tau)^{2}}+\frac{2 \pi n^{2} \sigma^{3}}{15} \int_{0}^{\infty} d r r^{3} \frac{\partial \Phi(|\mathbf{r}|)}{\partial r} \times$

ISSN 2071-0194. Ukr. J. Phys. 2011. Vol. 56, No. 8 
$\times \int_{0}^{r} G_{1}\left(r, r_{1}, \omega\right) \frac{\partial g_{0}\left(r_{1}\right)}{\partial r_{1}} r_{1} d r_{1}$

where

$G_{1}\left(r, r_{1}, \omega\right)=\frac{\tau_{0}}{\alpha}\left[\left(\sin \varphi_{1}-\cos \varphi_{1}\right) e^{-\varphi_{1}}-\right.$

$\left.-\left(\sin \varphi_{2}-\cos \varphi_{2}\right) e^{-\varphi_{2}}\right], \quad \varphi_{1,2}=\frac{\alpha}{2}\left(r \mp r_{1}\right)$,

$\alpha=\left(2 \omega \tau_{0}\right)^{1 / 2}$

The first term $\eta_{s}(\omega)$ in (7) allows for the contribution of the translational relaxation, i.e. the relaxation of the viscous stress tensor in the momentum space, whose characteristic time is equal to $\tau=m /(2 \beta)$. The frequency dependence $\eta_{s}(\omega)$ is, however, described mainly by means of the frequency behavior of the function $G_{1}\left(r, r_{1}, \omega\right)$ in a wide frequency range. The asymptotic behavior of $\eta_{s}(\omega)$ at low frequencies is $\sim \omega^{1 / 2}$, which coincides with the results obtained by the molecular dynamics method [10-12]; at high frequencies, it is $\sim \omega^{-1}$, while the phenomenological relaxation theory yields the asymptotics $\sim \omega^{-2}$. The function $G_{1}\left(r, r_{1}, \omega\right)$ is an ana$\log$ of the density of the relaxation time spectrum $H(\tau)$, $k\left(\tau / \tau^{\prime}\right)$, and $g\left(\tau / \tau^{\prime}\right)$ in [2, 3], whose explicit form is described by formula (8). According to (8), the frequency dependence of $G_{1}\left(r, r_{1}, \omega\right)$ is determined in a complex way based on the continuous spectrum of structural relaxation times. An analog of the characteristic time $\tau_{q}$ found in [13] can be obtained only in the approximation of the exponential attenuation of relaxing flows.

\section{Determination of $\eta_{s}(\omega)$ in the Case of Exponential Attenuation of Flows}

We now consider the case where the equilibrium structure is restored according to the exponential law. For this purpose, the Smoluchowski relaxation term in Eq. (3) will be replaced by the relaxation term resulting in the exponential attenuation law:

$\omega_{0} \hat{L} n_{2}\left(\mathbf{q}_{1}, \mathbf{r}_{1}, t\right)=-\omega_{0}\left[n_{0}\left(\mathbf{q}_{1}, \mathbf{r}_{1}, t\right)-n_{2}^{(0)}(|\mathbf{r}|, r, T)\right]$

where $n_{2}^{(0)}[|\mathbf{r}|, n(|\mathbf{q}|, t), T(|\mathbf{q}|, t)]=n_{2}^{(0)}(|\mathbf{r}|)+$ $\left.\tilde{n}_{2}^{(}|\mathbf{r}|, n, T\right)$ is the locally equilibrium binary density, $n_{2}^{(0)}(|\mathbf{r}|)=n_{0}^{2} g_{0}(|\mathbf{r}|)$ is the equilibrium binary density, while

$\tilde{n}_{2}\left(|\mathbf{r}|, n\left(\mathbf{q}_{1}, t\right), T\left(\mathbf{q}_{1}, t\right)\right)=$

$=\left(\frac{\partial n_{2}^{0}(|\mathbf{r}|)}{\partial n}\right)_{T} n\left(\mathbf{q}_{1}, t\right)+\left(\frac{\partial n_{2}^{0}(|\mathbf{r}|)}{\partial T}\right)_{n} T\left(\mathbf{q}_{1}, t\right)$.

The use of the hydrodynamic equation in the local approximation with regard for Eqs. (9), (10), and (3) yields the following solution for the time Fourier transform of the function $n_{2}\left(\mathbf{q}_{1}, \mathbf{r}, t\right)$ :

$n_{2}(\mathbf{q}, \mathbf{r}, \omega)=n_{0}^{2} g_{0}(|\mathbf{r}|)-$

$-n_{0}^{2}\left[n\left(\frac{\partial g_{0}(|\mathbf{r}|)}{\partial n}\right)_{T}+\gamma T\left(\frac{\partial g_{0}(|\mathbf{r}|)}{\partial T}\right)_{n}\right] \nabla \mathbf{u}(\omega)+$

$+\frac{i \omega \tau_{0}}{1-i \omega \tau_{0}}\left[\varphi_{0}(r) \nabla \mathbf{u}(\omega)+\varphi^{\alpha \beta}(r)\left\{\frac{\partial u^{\alpha}(\omega)}{\partial q_{1}^{\beta}}\right\}\right]$

where $\mathbf{u}(\omega)$ stands for the Fourier transform of the translational vector,

$\varphi^{\alpha \beta}(r)=\frac{r^{\alpha} r^{\beta}-(1 / 3) r^{2} \delta^{\alpha \beta}}{r} \frac{g_{0}(|\mathbf{r}|)}{\partial r}$,

$\varphi_{0}(r)=\frac{r}{3} \frac{\partial g_{0}(|\mathbf{r}|)}{\partial r}-\left[n\left(\frac{\partial g_{0}(|\mathbf{r}|)}{\partial n}\right)_{T}+\gamma T\left(\frac{\partial g_{0}(|\mathbf{r}|)}{\partial T}\right)_{n}\right]$

$\nabla \mathbf{u}(\omega)=\operatorname{div} \mathbf{u}(\omega)$

$\left\{\frac{\partial u^{\alpha}(\omega)}{\partial q^{\beta}}\right\}=\frac{1}{2}\left(\frac{\partial u^{\alpha}}{\partial q^{\beta}}+\frac{\partial u^{\beta}}{\partial q^{\alpha}}+\frac{2}{3} \delta^{\alpha \beta} \operatorname{div} \mathbf{u}(\omega)\right)$.

Performing the time Fourier transformation in (1) with regard for (11), we obtain the following expression for the dynamic coefficient of shear viscosity $\eta_{s}(\omega)$ :

$\eta_{s}(\omega)=\frac{n k T \tau}{1+(\omega \tau)^{2}}+\frac{2 \pi}{15} \frac{n^{2} k T \sigma^{3} \tau_{0}}{1+\left(\omega \tau_{0}\right)^{2}} \times$

$\times \int_{0}^{\infty} \frac{\partial \stackrel{*}{\Phi}(|\mathbf{r}|)}{\partial r} \frac{\partial g_{0}(|\mathbf{r}|)}{\partial r} r^{4} d r$

where $\stackrel{*}{\Phi}(|\mathbf{r}|)=\Phi(|\mathbf{r}|) / k T$ is the reduced potential of intermolecular interaction. 


\begin{tabular}{|c|c|c|c|c|c|c|c|c|c|c|c|}
\hline \multicolumn{4}{|c|}{$T=86 \mathrm{~K}$} & \multicolumn{4}{|c|}{$T=90 \mathrm{~K}$} & \multicolumn{4}{|c|}{$T=100 \mathrm{~K}$} \\
\hline \multirow[b]{2}{*}{$\begin{array}{c}\rho, \\
\mathrm{kg} / \mathrm{m}^{3}\end{array}$} & \multicolumn{3}{|c|}{$\eta_{S}, \mathrm{mPa} \cdot \mathrm{s}$} & \multirow[b]{2}{*}{$\begin{array}{c}\rho, \\
\mathrm{kg} / \mathrm{m}^{3}\end{array}$} & \multicolumn{3}{|c|}{$\eta_{S}, \mathrm{mPa} \cdot \mathrm{s}$} & \multirow[b]{2}{*}{$\begin{array}{c}\rho \\
\mathrm{kg} / \mathrm{m}^{3}\end{array}$} & \multicolumn{3}{|c|}{$\eta_{S}, \mathrm{mPa} \cdot \mathrm{s}$} \\
\hline & [13] & $\begin{array}{c}\text { form. } \\
(7)\end{array}$ & $\begin{array}{c}\text { form. } \\
(12)\end{array}$ & & [13] & $\begin{array}{c}\text { form. } \\
(7)\end{array}$ & $\begin{array}{c}\text { form. } \\
(12)\end{array}$ & & {$[13]$} & $\begin{array}{c}\text { form. } \\
(7)\end{array}$ & $\begin{array}{r}\text { form. } \\
(12)\end{array}$ \\
\hline 1402 & 0.272 & 0.271 & 0.234 & 1377 & 0.235 & 0.236 & 0.203 & 1312 & 0.180 & 0.174 & 0.147 \\
\hline 1407 & 0.276 & 0.276 & 0.237 & 1383 & 0.239 & 0.242 & 0.206 & 1319 & 0.183 & 0.178 & 0.149 \\
\hline \multirow[t]{3}{*}{1419} & 0.283 & 0.289 & 0.243 & 1396 & 0.245 & 0.254 & 0.212 & 1334 & 0.189 & 0.188 & 0.155 \\
\hline & & & & 1405 & 0.250 & 0.263 & 0.217 & 1347 & 0.194 & 0.198 & 0.160 \\
\hline & & & & 1418 & 0.255 & 0.276 & 0.224 & 1362 & 0.199 & 0.209 & 0.166 \\
\hline
\end{tabular}

Expression (12) also describes the dynamic coefficient of shear viscosity in the case where the equilibrium structure of the liquid is restored according to the exponential law. It is worth noting that expression (12) represents an analog of formulas (6.30), (6.42) from [2] and (5.17) from [3] obtained within the phenomenological theory with the difference that the second term is determined by means of $\Phi(|\mathbf{r}|)$ and $g_{0}(|\mathbf{r}|)$, i.e. the equilibrium molecular parameters are considered known at some choice of the model of a liquid [15-17].

\section{Choice of a Model and Numerical Calculations}

In our previous work [9], we performed numerical calculations of $\eta_{s}(\omega)$ using formula (7) for liquid argon in a wide frequency range. However, all our calculations supposed the constant friction coefficient of a liquid $\beta \approx 2.85 \cdot 10^{-13} \mathrm{~kg} / \mathrm{s}$, i.e. its dependence on the thermodynamic state parameters was not taken into account, while the density dependence in the radial distribution function $g_{0}(|\mathbf{r}|)$ was neglected. We also restricted ourselves to the qualitative comparison of the obtained numerical results with experimental data.

The numerical calculation of $\eta_{s}(\omega)$ according to Eqs. (7) and (12) meets some difficulties, as the friction coefficient of the liquid $\beta$ cannot be determined in the framework of the considered theory. The right-hand sides of the kinetic equations describing their time irreversibility (in our case, the Fokker-Planck collision operator) describe the dissipative processes in liquids and include the friction coefficient. In order to improve the agreement between the theoretically calculated values and the experimental results obtained for the coefficients $\eta_{s}(\omega), \beta$, and the relaxation times of the stress tensor in the momentum $\tau$ and configuration $\tau_{0}$ spaces, one should choose the optimal forms of the potential of intermolecular interaction $\Phi(|\mathbf{r}|)$, the radial distribution function $g_{0}(|\mathbf{r}|)$, as well as their dependence on the density $\rho$ and the temperature $T$.

As the initial approximation, we use the model of $\Phi(|\mathbf{r}|), g_{0}(|\mathbf{r}|)$, and $\beta$ considered in $[18,19]$ in the following form:

$\Phi(|\mathbf{r}|)= \begin{cases}\infty, & \text { for } r<\sigma \\ 4 \varepsilon\left(r^{-12}-0.5 r^{-6}\right), & \text { for } r \geq \sigma\end{cases}$

$g(|\mathbf{r}|)=y(\stackrel{*}{\rho}) \exp \left(-\frac{\Phi(|\mathbf{r}|)}{k T}\right)$

$\beta^{2}=\frac{4 \pi}{3} \rho \sigma^{3} \int_{0}^{\infty} \nabla^{2} \Phi(|\mathbf{r}|) g_{0}(|\mathbf{r}|) r^{2} d r$

where $\varepsilon$ is the depth of the potential well, $y(\stackrel{*}{\rho})=$ $(2-\stackrel{*}{\rho}) / 2(1-\stackrel{*}{\rho})^{3}$ is the Carnahan-Starling contact function [17], $\stackrel{*}{\rho}=\frac{\pi}{6} \frac{N_{0} \sigma^{3}}{M} \rho$ is the reduced density, $N_{0}$ is the Avogadro number, $M$ is the molar mass, $\rho=m n$ is the liquid density, $m, \sigma$, and $n=N / V$ are, respectively, the mass, diameter, and density of liquid particles, and $\nabla^{2}=\left(1 / r^{2}\right)(\partial / \partial r)\left(r^{2} \partial / \partial r\right)$ is the radial part of the Laplace operator.

Based on expressions (7) and (12) with regard for (13), we performed numerical calculations of the dynamic coefficient of shear viscosity $\eta_{s}(\omega)$ for liquid argon as a function of the thermodynamic state parameters $(\rho, T)$ in a wide frequency range. The corresponding values of $\rho$ and $T$ are taken from experimental studies $[13,14]$.

The results of numerical calculations of the isofrequency coefficient $\eta_{s}(\omega)$ at $\stackrel{*}{\nu}=10^{-6}\left(\nu \approx 10^{7} \mathrm{~Hz}\right)$ performed with the use of formulas (7) and (12) in the temperature interval $84 \leq T \leq 100 \mathrm{~K}$ at various densities $\rho$ for liquid argon and their comparison with experimental data $[13,14]$ are given in Tables 1 and 2 . 


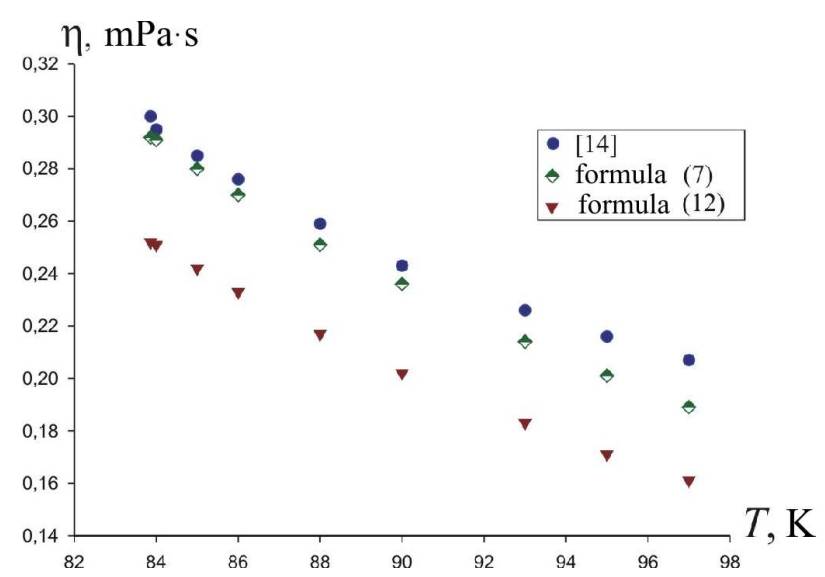

Fig. 1. Temperature dependence of the shear viscosity $\eta_{s}(\omega)$ at $\stackrel{*}{\nu}=10^{-6}\left(\nu \sim 10^{7} \mathrm{~Hz}\right)$ and comparison with experimental results [14]

One can see that the theoretical values of $\eta_{s}(\omega)$ are in good quantitative agreement with the experimental data as concerns both the temperature and the density dependences. The values of $\eta_{s}(\omega)$ calculated by formula (12) are somewhat underestimated.

The temperature dependence of the isofrequency $(\stackrel{*}{\nu}=$ $\left.10^{-6}, \nu \sim 10^{7} \mathrm{~Hz}\right)$ shear viscosity $\eta_{s}(\omega)$ is compared with experimental results [14] in Fig. 1. Figure 2 presents the frequency dependence $\eta_{s}(\omega)$ at $T_{1}=84 \mathrm{~K}$ and $T_{2}=90$ $\mathrm{K}$ in the frequency range $10^{-4} \leq \stackrel{*}{\nu}^{*} 10\left(10^{8} \leq \nu \leq 10^{13}\right.$ $\mathrm{Hz}$ ) obtained using formulas (7) and (12).

The frequency dispersion region of $\eta_{s}(\omega)$ obtained on the basis of the diffusion mechanism (i.e. formula (7)) appears wide $\sim 10^{5} \mathrm{~Hz}$, which agrees with experimental conclusions about the contribution of the structural relaxation to the viscous properties of liquids [2]. At the same time, the frequency dispersion region of $\eta_{s}(\omega)$ found using the exponential attenuation law for the viscous stress tensor (formula (12)) is narrow $\sim 10^{2} \mathrm{~Hz}$,

\begin{tabular}{c|c|c|c|c}
$\mathbf{T} \mathbf{a} \mathbf{b} \mathbf{l} \mathbf{~ e ~} \mathbf{2}$ \\
\hline \multirow{2}{*}{$T, \mathrm{~K}$} & $\rho, \mathrm{kg} / \mathrm{m}^{3}$ & \multicolumn{3}{|c}{$\eta_{S}, \mathrm{mPa} \cdot \mathrm{s}$} \\
\cline { 3 - 5 } & & {$[14]$} & form. $(7)$ & form. $(12)$ \\
\hline 83,86 & 1414 & 0.300 & 0.292 & 0.252 \\
84 & 1413.5 & 0.295 & 0.291 & 0.251 \\
85 & 1407 & 0.285 & 0.280 & 0.242 \\
86 & 1401 & 0.276 & 0.270 & 0.233 \\
88 & 1388 & 0.259 & 0.251 & 0.217 \\
90 & 1376 & 0.243 & 0.236 & 0.202 \\
93 & 1357 & 0.226 & 0.214 & 0.183 \\
95 & 1344 & 0.216 & 0.201 & 0.171 \\
97 & 1331 & 0.207 & 0.189 & 0.161 \\
\hline
\end{tabular}

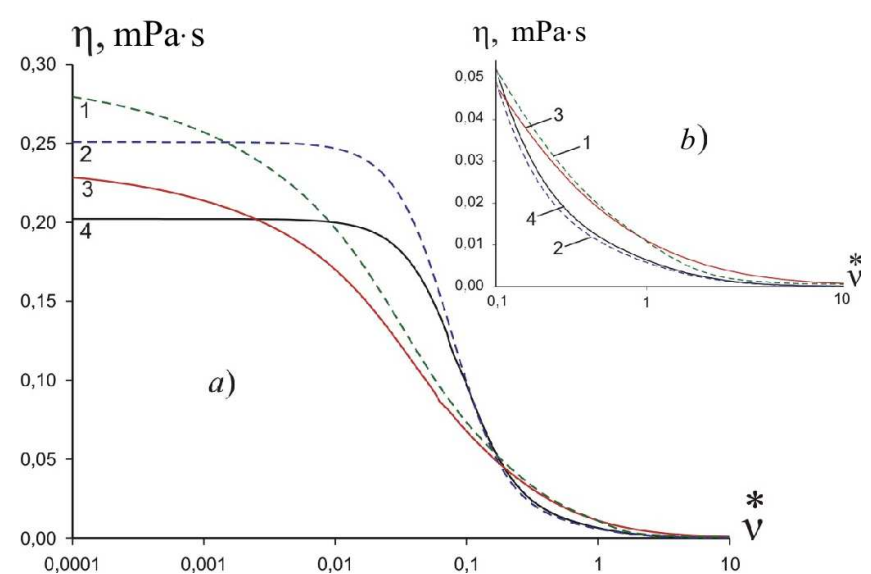

Fig. 2. Frequency dependence of the shear viscosity $\eta_{s}(\omega)$ at $T=$ $84 \mathrm{~K}$ (dashed curve) and $T=90 \mathrm{~K}$ (solid curve). Numerical results are obtained according to formula (7) 1,3 and formula (12) 2,4

which agrees with both experimental acoustic and theoretical results obtained on the basis of the phenomenological theory $[2,3]$. This difference is evidently due to the fact that, at low frequencies, there exists the asymptotics $\sim \omega^{1 / 2}$ (according to (7)), and the frequency dispersion starts early. At high frequencies, $\eta_{s}(\omega)$ attenuates according to the slower law $\sim \omega^{-1}$. As follows from Eq.(12), however, the shear viscosity at low frequencies is static. Whereas $\eta_{s}(\omega) \sim \omega^{-2}$ at high frequencies, which coincides with the results of formulas (6.30) and (6.42) in [2]. Consequently, the determination of the frequency dispersion region of the shear viscosity coefficient is closely related to the mechanism of dispersal of internal relaxing flows in liquids.

1. M. Kornfeld, Elasticity and Strength of Liquids (GITTL, Moscow, 1951) (in Russian).

2. I.G. Mikhailov, V.A. Solov'ev, and Yu.P. Syrnikov, Fundamentals of Molecular Acoustics (Nauka, Moscow, 1964) (in Russian).

3. Physical Acoustics: Principles and Methods. Vol. 2, pt. A. Properties of Gases, Liquids, and Solutions, edited by W. Mason (Academic Press, New York, 1965).

4. B.J. Alder and W.E. Alley, Phys. Today, 37, 56 (1984).

5. S. Odinaev and A.A. Adkhamov, Molecular Theory of Structural Relaxation and Transport Phenomena in Liquids (Donish, Dushanbe, 1998) (in Russian).

6. M.H. Ernst, E.H. Hauge, and M.J. van Leeuwen, Phys. Rev. Lett. 25, 1254 (1970).

7. I.Z. Fisher, Zh. Eksp. Teor. Fiz. 61, 1647 (1971). 
8. Y. Pomeau, Phys. Rev. A 5, 2569 (1972); 7, 1134 (1973).

9. A.A. Adkhamov and S. Odinaev, Ukr. Fiz. Zh. 29, 1517 (1984).

10. A.N. Lagar'kov and V.M. Sergeev, Usp. Fiz. Nauk 125, 409 (1978).

11. D.J. Evans, H.J.M. Hanley, and S. Hess, Phys. Today, 37, 26 (1984).

12. D.J. Evans and G.P. Morris, Statistical Mechanics of Nonequilibrium Liquids (Academic Press, London, 1990).

13. S.A. Mikhailenko, B.G. Dudar', and V.A. Shmidt, Fiz. Nizk. Temp. 1, 224 (1975).

14. P. Malbrunot, A. Boyer, and E. Charles, Phys. Rev. A 27, 1523 (1983).

15. J.O. Hirschfelder, Ch.F. Curtiss, and R.B. Bird, The Molecular Theory of Gases and Liquids (Wiley, New York, 1964).

16. Physics of Simple Liquids, edited by H.N.V. Temperley, J.S. Rowlinson, and G.S. Rushbroooke (North-Holland, Amsterdam, 1969).

17. I.R. Yukhnovskii and M.F. Golovko, Statistical Theory of Classical Equilibrium Systems (Naukova Dumka, Kiev, 1980) (in Russian).

18. S. Odinaev, D. Akdodov, and Kh. Mirzoaminov, Dokl. Akad. Nauk Resp. Tajik. 52, 928 (2009).
19. S. Odinaev and Kh. Mirzoaminov, Ukr. Fiz. Zh. 551103 (2010).

Received 07.07.10

Translated from Russian by H.G. Kalyuzhna

ПРО ВИЗНАЧЕННЯ ОБЛАСТІ ЧАСТОТНОЇ ДИСПЕРСІЇ КОЕФІЦІЄНТІВ ПЕРЕНЕСЕННЯ КЛАСИЧНИХ РІДИН

ЗАЛЕЖНО ВІД ПРИРОДИ ЗАТУХАННЯ

РЕЛАКСУЮЧИХ ПОТОКІВ

C. Одінаєе

P е $з$ ю $\mathrm{M}$

Розглянуто область частотної дисперсії динамічного коефіцієнта зсувної в'язкості $\eta_{s}(\omega)$ простих рідин, що отриманий методом кінетичних рівнянь, коли відновлення рівноважної структури рідини відбувається за законом дифузії або експоненціально. За певного вибору потенціалу міжмолекулярної взаємодії $\Phi(|\mathbf{r}|)$ і рівноважної радіальної функції розподілу $g_{0}(|\mathbf{r}|)$ проведено числові розрахунки $\eta_{s}(\omega)$ для рідкого аргону залежно від густини $\rho$, температури $T$ та частоти $\omega$. Теоретично обчислені результати для зсувної в'язкості $\eta_{s}(\omega)$ добре узгоджуються $з$ експериментальними даними. Показано, що область частотної дисперсії $\eta_{s}(\omega)$ на основі дифузійного механізму, тобто структурної релаксації, широка $\sim 10^{5}$ Гц, а у випадку експоненційного затухання в'язкого тензора напруги - вузька $\sim 10^{2}$ Гц, що відповідає як акустичним вимірам, так і результатам феноменологічної теорії. 